\title{
Early systemic sclerosis: marker autoantibodies and videocapillaroscopy patterns are each associated with distinct clinical, functional and cellular activation markers
}

Gabriele Valentini ${ }^{1 *}$, Antonella Marcoccia ${ }^{2}$, Giovanna Cuomo', Serena Vettori', Michele ludici', Francesco Bondanini ${ }^{3}$, Carlo Santoriello ${ }^{4}$, Aldo Ciani ${ }^{5}$, Domenico Cozzolino $^{6}$, Giovanni Maria De Matteis ${ }^{7}$, Salvatore Cappabianca ${ }^{8}$, Filiberto Vitelli ${ }^{9}$ and Alberto Spanò ${ }^{3}$

\begin{abstract}
Introduction: Early systemic sclerosis (SSc) is characterized by Raynaud's phenomenon together with scleroderma marker autoantibodies and/or a scleroderma pattern at capillaroscopy and no other distinctive feature of SSc. Patients presenting with marker autoantibodies plus a capillaroscopic scleroderma pattern seem to evolve into definite SSc more frequently than patients with either feature. Whether early SSc patients with only marker autoantibodies or capillaroscopic positivity differ in any aspect at presentation is unclear.
\end{abstract}

Methods: Seventy-one consecutive early SSc patients were investigated for preclinical cardiopulmonary alterations. Out of these, 44 patients and 25 controls affected by osteoarthritis or primary fibromyalgia syndrome were also investigated for serum markers of fibroblast (carboxyterminal propeptide of collagen I), endothelial (soluble E-selectin) and T-cell (soluble IL-2 receptor alpha) activation.

Results: Thirty-two of the 71 patients (45.1\%) had both a marker autoantibody and a capillaroscopic scleroderma pattern (subset 1), 16 patients (22.5\%) had only a marker autoantibody (subset 2), and 23 patients (32.4\%) had only a capillaroscopic scleroderma pattern (subset 3). Patients with marker autoantibodies ( $n=48,67.6 \%$ ) had a higher prevalence of impaired diffusing lung capacity for carbon monoxide $(P=0.0217)$ and increased serum levels of carboxyterminal propeptide of collagen I $(P=0.0037)$, regardless of capillaroscopic alterations. Patients with a capillaroscopic scleroderma pattern $(n=55,77.5 \%)$ had a higher prevalence of puffy fingers $(P=0.0001)$ and increased serum levels of soluble E-selectin $(P=0.0003)$ regardless of marker autoantibodies.

Conclusion: These results suggest that the autoantibody and microvascular patterns in early SSc may each be related to different clinical-preclinical features and circulating activation markers at presentation. Longitudinal studies are warranted to investigate whether these subsets undergo a different disease course over time.

Keywords: Raynaud's phenomenon, early systemic sclerosis, systemic sclerosis marker autoantibodies, nailfold videocapillaroscopy, preclinical organ involvement, puffy fingerscirculating activation markers, carboxyterminal propeptide of collagen I, soluble E-selectin, soluble IL-2 receptor alpha

\footnotetext{
* Correspondence: gabriele.valentini@unina2.it

'Rheumatology Unit, Second University of Naples, via Pansini 5, 80131

Naples, Italy

Full list of author information is available at the end of the article
} 


\section{Introduction}

Early systemic sclerosis (SSc) is a condition characterized by Raynaud's phenomenon (RP) associated with SSc marker autoantibodies (anti-Scl-70, anticentromere antibodies, anti-RNA polymerase III, anti-fibrillarin, anti-PmScl, and anti-Th/To) and/or nailfold videocapillaroscopy (NVC) findings typical of SSc (namely, megacapillaries and avascular areas - commonly referred to as an 'NVC scleroderma pattern'). By definition, patients with early SSc do not show any distinctive clinical manifestation of the disease (namely, sclerodactyly, digital ulcers/scars, two or more teleangectasias, clinically visible nailfold capillaries, cutaneous calcinosis, X-ray bibasilar lung fibrosis, X-ray esophageal dysmotility, electrocardiographic signs of myocardial fibrosis, or a serum creatinine increase suggestive of scleroderma renal crisis) except puffy fingers and/or arthritis [1-4].

Various attempts have been made to find a correct classification for these patients who do not meet the preliminary 1990 American College of Rheumatology (ACR) SSc criteria [5] in order to foster their inclusion in clinical trials. In fact, in 2001 LeRoy and Medsger proposed that RP patients with SSc marker autoantibodies or an NVC scleroderma pattern should be classified as being affected by limited SSc [1]. Seven years later, Koenig and colleagues validated the LeRoy and Medsger criteria in a large 20 -year prospective study [2]. They found that, at the last follow-up, patients presenting with SSc marker autoantibodies and a scleroderma pattern at capillaroscopy (referred to as subset 1 in the present paper), with no other manifestation distinctive of the disease other than puffy fingers and/or arthritis, had developed definite SSc 60 times more frequently than patients presenting with only RP. Patients with either only SSc marker autoantibodies (subset 2) or a capillaroscopic scleroderma pattern (subset 3) had developed definite SSc five and eight times more frequently, respectively, than patients presenting with only RP. Koenig and colleagues therefore suggested that such cases be labeled collectively as early SSc.

Two years ago, we reported that $42 \%$ of thus defined early SSc patients admitted to our tertiary Rheumatology Unit had preclinical functional alterations of the esophagus, lung or heart [3]. Subsequently, we showed that a high percentage of early SSc patients develop definite SSc within 5 years of presentation and found that some circulating fibroblast, endothelial and T-cell activation markers could be used as predictors of disease evolution besides marker autoantibodies and NVC findings [4]. In their study, Koenig and colleagues did not systematically investigate visceral involvement and neither did they consider circulating activation markers [2]. In our previous papers we were unable to try any subsetting among our early SSc patients because of the low number of early SSc patients with only an NVC scleroderma pattern and negative for SSc marker autoantibodies admitted to our unit $[3,4]$.

In the present study, we evaluated whether clinical, preclinical functional or cellular activation markers are related to distinct early SSc subsets at presentation by merging our early SSc cohort with that recruited at a secondary Angiology Unit, where a significant number of patients with early SSc were SSc marker antibodynegative and capillaroscopy-positive.

\section{Materials and methods \\ Patients}

Patients admitted for evaluation of RP from 1 November 2000 to 30 June 2012 to the outpatient clinic of the Rheumatology Unit of the Second University of Naples and from 1 January 2008 to 30 June 2012 to the outpatient clinic of the Angiology Unit of the Pertini Hospital in Rome who met the Koenig and colleagues criteria for early SSc (that is, RP with SSc marker autoantibodies and/or an NVC scleroderma pattern without any clinical manifestation of definite SSc) [2] were enrolled in the study after giving written informed consent.

The diagnosis of RP was confirmed if patients fulfilled LeRoy and Medsger's criteria [6]; that is, if a cold challenge induced bilateral, episodic biphasic or triphasic (pallor followed by dusky blueness and/or redness) color changes of fingers. According to standard clinical practice, and for the purpose of a correct disease classification, patients underwent a complete screening workplan.

\section{Routine assessment}

Each patient was investigated to identify any feature that precluded inclusion in the study. In detail, we looked for the following features: dysphagia, effort dyspnea, and findings consistent with previous scleroderma renal crisis at history; symmetrical skin sclerosis, digital ulcers/scars, two or more teleangectasias, clinically visible nailfold capillaries, cutaneous calcinosis, and recent-onset accelerated or malignant arterial hypertension at physical examination; bibasilar lung fibrosis at chest X-ray scan; esophageal dysmotility at barium esophageal X-ray scan; and blocks and/ or $\mathrm{Q}$ waves at electrocardiography. In addition, blood cell count, urinalysis, blood urea nitrogen, serum creatinine, alanine aminotransferase, aspartate aminotransferase, erythrosedimentation rate, serum protein electrophoresis with the evaluation of $\gamma$-globulin concentration, and serum $\mathrm{C} 3$ and $\mathrm{C} 4$ concentration were also evaluated.

\section{Nailfold videocapillaroscopy assessment}

NVC was carried out with an optical probe videocapillaroscope equipped with a $\times 200$ magnification contact lens and connected to image analysis software (Videocap; DS MediGroup, Milan, Italy). The nailfold of the 
second, third, fourth and fifth fingers was examined bilaterally in each patient. Four consecutive fields extending over $1 \mathrm{~mm}$ in the middle of the nailfold were studied per finger. All images of patients from both centers were stored and reviewed by a physician (MI) experienced in NVC [3,4,7]. The degree of capillary enlargement (on a scale of 0 to 3 ) and capillary loss (graded A to D) were investigated. Megacapillaries (capillary enlargement $\geq 2$ ) and/or avascular areas (capillary loss grade $\geq C$ ) were considered a scleroderma pattern [8,9]. Moreover, since NVC active and late patterns, which are characterized by a lower capillary density, have been found to be associated with more severe disease in SSc patients [10], we also subdivided patients according to the mean capillary number per millimeter; that is, $>9 / \mathrm{mm}, 9$ to $7 / \mathrm{mm}$, 6 to $4 / \mathrm{mm}$, and $\leq 3 / \mathrm{mm}$.

\section{Autoantibody profile assessment}

An autoantibody screening and profiling of sera collected at the first visit was performed. Antinuclear antibodies and anticentromere antibodies were searched for by a conventional indirect immunofluorescence assay on HEp2 cells, using fluorescent human $\gamma$-globulins as detection antibodies and a serum dilution of 1:160 as the cutoff value (Astra srl, Pavone Canavese, Italy). Anti-double-stranded DNA antibodies were also searched for by indirect immunofluorescence on Crithidia Luciliae, using fluorescent human $\gamma$-globulins as detection antibodies and a serum dilution of 1:40 as the cutoff value (Astra srl). Anti-Scl-70, anti-PmScl, anti-SSA, anti-SSB, anti-Sm, anti-Jo1, and anti-U1RNP antibodies were identified by commercially available ELISA kits (Chematil srl, Angri, Italy), using $25 \mathrm{U} / \mathrm{ml}$ as the cutoff value. Anti-RNA polymerase III and anti-fibrillarin antibodies were identified by EliA Varelisa test (Phadia, Freiburg, Germany), using $150 \mathrm{U} / \mathrm{ml}$ as the cutoff value. Anti Th/To were identified by western blotting (reagents by Arnika srl, Milano, Italy). To avoid a differential verification bias, sera were blindly exchanged between the two centers and only confirmed results were considered. Each result was confirmed.

\section{Assessment of preclinical internal organ involvement}

Patients from both centers underwent B-mode echocardiography and lung function study. Echocardiographic examination was performed as reported elsewhere [11]. The detection of diastolic abnormalities at B-mode echocardiography, indicated by an inverted ratio between early/ (atrial) late ventricular filling velocity $(\mathrm{E} / \mathrm{A}$ ratio $<1$ ), in the absence of arterial hypertension, coronary artery disease and other symptoms/signs of cardiac disease, was regarded as early scleroderma heart involvement [12]. The detection of a diffusing lung capacity for carbon monoxide (DLCO) or a forced vital capacity $<80 \%$ of the predicted values in the absence of a smoking habit and/or obstructive lung disease at lung function study was regarded as SSc lung involvement $[13,14]$. Moreover, after further informed consent, patients from the Rheumatology Unit with a reduced DLCO underwent high-resolution computed tomography of the chest [15].

\section{Fibroblast, endothelial and T-cell activation markers}

Early SSc patients for whom a baseline serum specimen was available and 25 controls, matched for sex and age and affected by osteoarthritis or primary fibromyalgia syndrome, were investigated at the Rheumatology Unit laboratory for the fibroblast, endothelial and T-cell activation markers carboxyterminal telopeptide of type I collagen (ICTP), soluble E-selectin (sE-selectin) and soluble IL-2 receptor alpha (sIL-2R $\alpha$ ), as described previously [4]. Briefly, sIL-2R $\alpha$ and sE-selectin concentrations were measured by a multiplex suspension immunoassay (expressed as $\mathrm{pg} / \mathrm{ml}$ ), based on the use of spectrally encoded beads, each coupled with a capture antibody specific to the analyte of interest, as the solid support and a biotinylated detection antibody-streptavidine-phycoerythrin complex as the reporter system (all reagents were from Merk Millipore, Billerica, MA, USA) to be read by a double laser-based fluorimetric instrument (Luminex 200; Luminex Corporation, Austin, TX, USA). The ICTP concentrations were measured by a conventional competitive radioimmune assay (expressed as $\mu \mathrm{g} / \mathrm{l}$ ) (UniQ kits; Orion Diagnostica, Espoo, Finland). We did not measure the aminoterminal propeptide of type III collagen serum concentration because in our previous study it did not differ between early SSc and either undifferentiated connective tissue disease patients or controls at baseline [4].

Based on the autoantibody and capillaroscopic profiles, early SSc patients were divided into three subsets: subset 1, patients with both SSc marker autoantibody positivity and an NVC scleroderma pattern; subset 2, patients with only SSc marker autoantibody positivity; and subset 3, patients with only an NVC scleroderma pattern.

The study was reviewed and approved by the Azienda Ospedaliera Universitaria Seconda Università degli Studi di Napoli Ethics Committee and by the ASL RM/B Ethics Committee.

\section{Statistical analysis}

GraphPad Prism software version 6.0 (GraphPad Software Inc., San Diego, CA, USA) was used for statistical analysis. Continuous data were expressed as the mean \pm standard deviation and the median with range, and were compared by the unpaired Student's $t$ test or Mann-Whitney U test as appropriate when two groups were analyzed, and by one-way analysis of variance or Kruskal-Wallis test as appropriate when three or more groups were compared. 
Categorical data were analyzed by Fisher's exact test and the chi-square test when two or three groups, respectively, were considered.

\section{Results}

Forty-five patients were enrolled at the Rheumatology Unit of the Second University of Naples and 26 patients at the Angiology Unit of the Pertini Hospital in Rome. Table 1 presents the main epidemiologic, laboratory and capillaroscopic features of the patients enrolled by the Rheumatology and Angiology Units. There was no difference between the two series in terms of age, sex and time interval elapsed from the onset of RP. However, the prevalence of subset 1 patients was significantly higher (26/45 (57.8\%) vs. 6/26 (23.1\%), $P=0.0098$ ) and the prevalence of subset 3 patients significantly lower $(3 / 45(6.7 \%)$ vs. $20 / 26$ (76.9\%), $P=$ $0.0001)$ in the Rheumatology series. Subset 2 patients were recruited only by the Rheumatology Unit.

By definition, no patient presented any clinical feature of SSc other than puffy fingers or arthritis. The prevalence of puffy fingers was significantly higher in both subset 1 and subset 3 Angiology Unit patients than in the respective Rheumatology Unit patients (6/6 vs. $4 / 26, P=0.004$ for subset $1 ; 19 / 20$ vs. $1 / 3, P=0.04$ for subset 3$)$. The prevalence of arthritis did not differ statistically between the two centers $(1 / 26(3.85 \%)$ for Rheumatology Unit patients vs. $2 / 6$ (33.3\%) for Angiology Unit patients, $P>0.05$ for subset $1 ; 0 / 3$ (0\%) for Rheumatology Unit patients vs. $2 / 20$ (10\%) for Angiology Unit patients, $P>0.05$ for subset 3 ). Because of the low number of subset 3 patients recruited at the Rheumatology Unit and of subset 1 patients admitted to the Angiology Unit, in order to increase the power of the analysis we merged the two series.
Figure 1 shows the prevalence of puffy fingers (Figure 1A) and arthritis (Figure 1B) in the three subsets. The prevalence of puffy fingers was significantly higher in subset 3 patients $(20 / 23,87 \%)$ from the pooled series than in subset $1(10 / 32,31.3 \%)$ and subset $2(2 / 16,12.5 \%)$ patients $(P=$ $0.0001)$ (Figure 1A). Thirty out of the 32 (93.75\%) patients across the three subsets with puffy fingers had a positive NVC, and 25/39 (64.1\%) patients without puffy fingers had a positive NVC $(P=0.0037)$ (Figure $1 C)$.

The prevalence of functional abnormalities did not differ between each subset of patients from the two centers. A forced vital capacity $<80 \%$ of the predicted value and an inverted E/A ratio were found only in 2/32 (6.25\%) and $1 / 32(3.13 \%)$ subset 1 patients, respectively, and in no subset 2 and subset 3 patients. However, an impaired DLCO was more prevalent in subset $1(11 / 32$, $34.4 \%)$ and subset $2(6 / 16,37.5 \%)$ patients than in subset $3(2 / 23,8.7 \%)$ patients $(P=0.057)$ (Figure $2 \mathrm{~A})$. In addition, out of the 48 marker autoantibody-positive patients (that is, subset $1+$ subset 2 ), DLCO $<80 \%$ of the predicted value was detected in 17 (35.4\%) versus $2 / 23(8.7 \%)$ marker autoantibody-negative subset 3 patients $(P=0.0217)$ (Figure $2 \mathrm{~B})$. Lastly, 6/14 (42.9\%) patients with SSc marker autoantibodies other than anticentromere antibodies (three anti-Scl-70; three antiRNA polymerase III) had a reduced DLCO as compared with $11 / 34(34.4 \%)$ anticentromere antibody-positive patients. The difference did not reach statistical significance (Figure 2C).

A ground glass opacity involving $<5 \%$ of inferior lobes and an interlobular septal thickening were found in two patients and two different patients, respectively, by lung high-resolution computed tomography.

Table 1 Epidemiologic, laboratory and capillaroscopic features of the two early systemic sclerosis series

\begin{tabular}{|c|c|c|c|}
\hline Feature & Rheumatology Unit $(n=45)$ & Angiology Unit $(n=26)$ & $P$ value \\
\hline Sex (female/male ratio) & $43 / 2$ & $22 / 4$ & NS \\
\hline Age (years) & $41(17-73)$ & $37.5(16-71)$ & NS \\
\hline RP duration (years) & $3(0.5-24)$ & $3(1-20)$ & NS \\
\hline Subset 1: antibody-positive, NVC-positive & $26(57.8)$ & $6(2.2)$ & 0.0098 \\
\hline Anti-Scl-70 antibody-positive & $5(19.2)$ & 0 & \\
\hline ACA-positive & $19(73.1)$ & $3(50)$ & \\
\hline Anti-RNA polymerase III antibody-positive & $2(7.7)$ & $3(50)$ & \\
\hline Megacapillaries only & $21(80.8)$ & $3(50)$ & \\
\hline Avascular areas \pm megacapillaries & $5(19.2)$ & $3(50)$ & \\
\hline Subset 2: antibody-positive, NVC-negative & $16(35.6)$ & 0 & - \\
\hline AntiScl-70 antibody-positive & $4(25)$ & 0 & \\
\hline ACA-positive & $12(75)$ & 0 & \\
\hline Anti-RNA polymerase III antibody-positive & 0 & 0 & \\
\hline Subset 3: antibody-negative, NVC-positive & $3(6.7)$ & $20(76.9)$ & 0.0001 \\
\hline Megacapillaries only & $3(100)$ & $20(100)$ & - \\
\hline Avascular areas \pm megacapillaries & 0 & 0 & - \\
\hline
\end{tabular}

All data expressed as median (range) or number (percentage). ACA, anticentromere antibody; NVC, nailfold videocapillaroscopy; RP, Raynaud's phenomenon. 


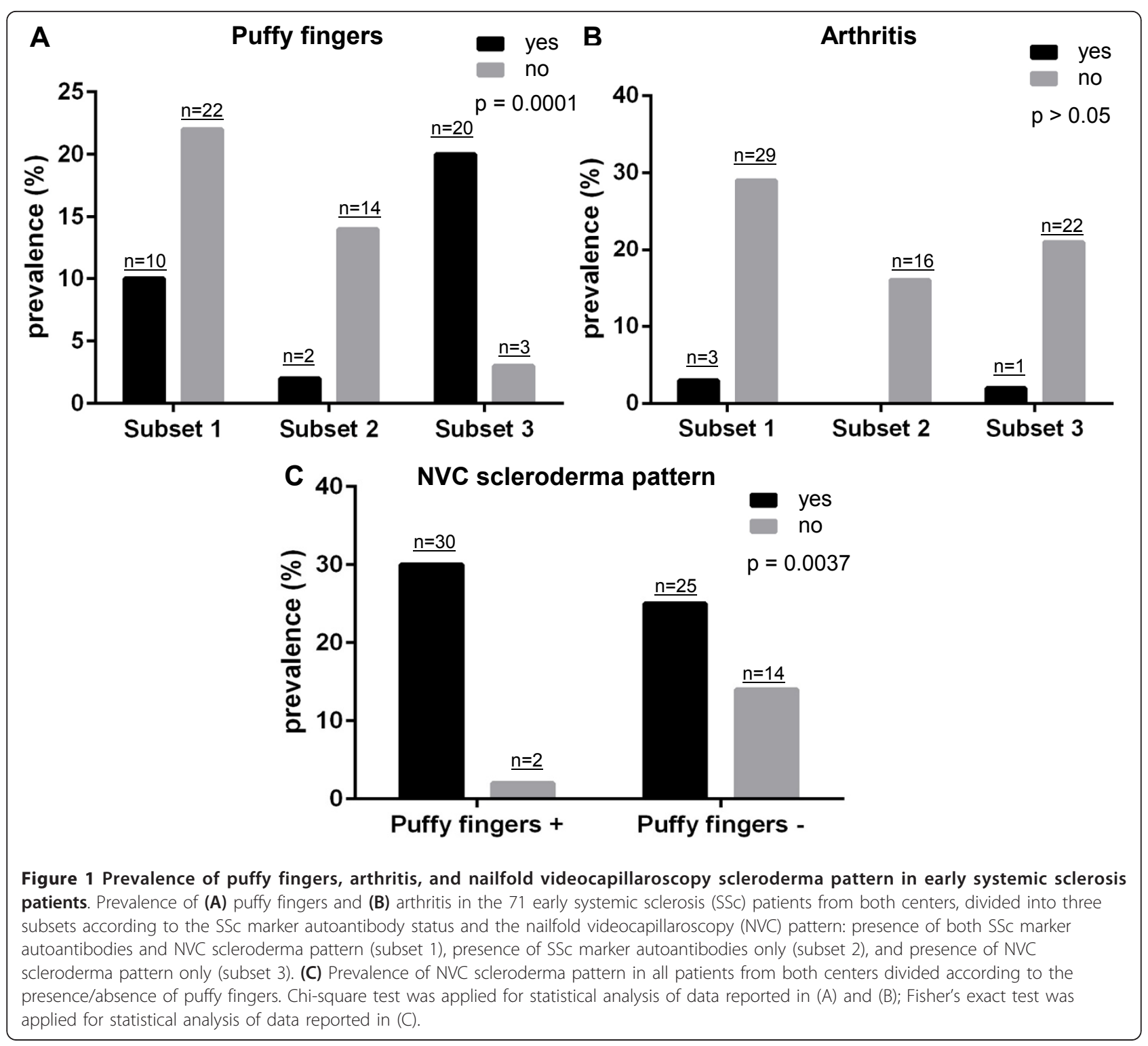

Sera collected at admission were available for 22/45 (48.9\%) patients from the Rheumatology Unit and for 22/26 (84.6\%) patients from the Angiology Unit. The clinical, serological and NVC features of the 22 Rheumatology Unit patients reflected the characteristics of the whole series of the center (data not shown). Figure 3 shows serum levels of the investigated activation markers in the three early SSc subsets and in controls. Two main findings emerged from this analysis. Firstly, serum concentrations of ICTP were significantly higher in patients with SSc marker autoantibodies (median $=4.025 \mu \mathrm{g} / \mathrm{l}$ in subset $1+$ subset 2 , range 1.883 to $10.53 \mu \mathrm{g} / \mathrm{l}$ ) than in subset 3 patients $($ median $=2.51 \mu \mathrm{g} / \mathrm{l}$, range 1.55 to $6.972 \mu \mathrm{g} / \mathrm{l} ; P=0.0108)$ (Figure 3A). In detail, $4 / 15$ (26.7\%) subset 1 patients and $3 /$ 9 (33.3\%) subset 2 patients had ICTP levels that exceeded the 95 th percentile $(5.05 \mu \mathrm{g} / \mathrm{l})$ of the values measured in controls with respect to $2 / 17$ (11.8\%) subset 3 patients. Secondly, levels of sE-selectin were significantly higher in patients with an NVC scleroderma pattern (median = $1.031 \mathrm{pg} / \mathrm{ml}$ in subset $1+$ subset 3 , range 0.425 to $7.51 \mathrm{pg} /$ $\mathrm{ml}$ ) than in subset 2 patients with only SSc marker autoantibodies (median $=0.67 \mathrm{pg} / \mathrm{ml}$, range 0.486 to $1.203 \mathrm{pg} / \mathrm{ml}$; $P=0.033$ ) (Figure 3B). In detail, sE-selectin levels exceeded the 95 th percentile of the values recorded in controls $(1.709 \mathrm{pg} / \mathrm{ml})$, in $11 / 17(64.7 \%)$ subset 3 patients, in $2 / 15$ (13.3\%) subset 1 patients and in $0 / 9$ subset 2 patients $(P=$ $0.0003)$. Noteworthy, we found that among all three subsets $\mathrm{sE}$-selectin levels were significantly higher in patients with puffy fingers than in those without puffy fingers ( $2.013 \mathrm{pg} / \mathrm{ml}$, range 0.55 to 7.51 vs. $0.725 \mathrm{pg} / \mathrm{ml}$, range 0.425 to $1.203 \mathrm{pg} / \mathrm{ml} ; P<0.0001$ ) (Figure $3 C$ ). Finally, sIL$2 \mathrm{R} \alpha$ levels were lower in SSc marker autoantibody-negative 


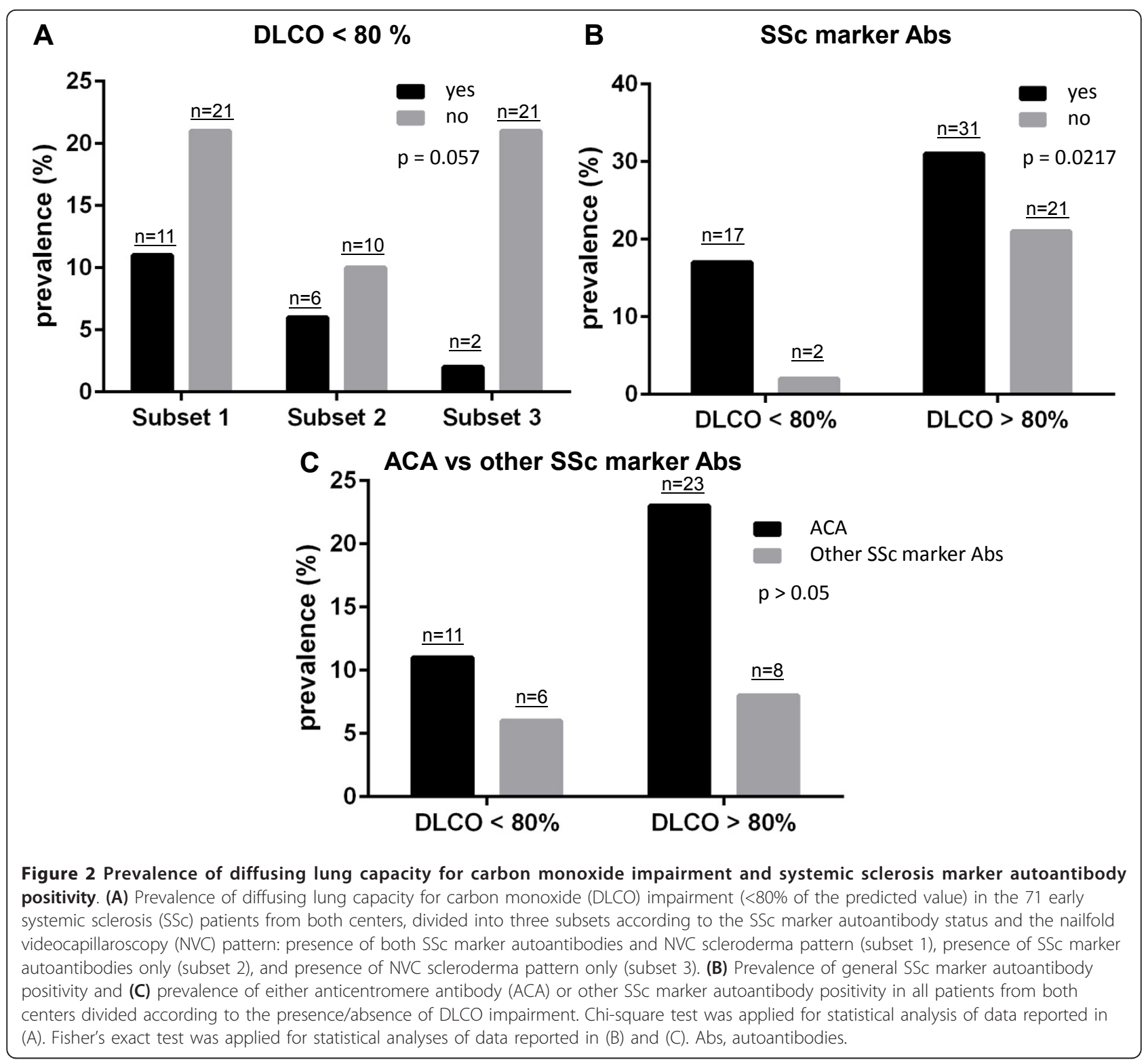

patients (subset 3: median $=24.41 \mathrm{pg} / \mathrm{ml}$, range 0 to 411.2 ) than in SSc marker autoantibody-positive patients (subset $1+$ subset 2 patients: median $=239.8 \mathrm{pg} / \mathrm{ml}$, range 66.74 to $1,003 \mathrm{pg} / \mathrm{ml} ; P=0.0015$ ) (Figure $3 \mathrm{D}$ ). sIL-2R $\alpha$ levels were lower than the 5 th percentile of control values $(25.7 \mathrm{pg} / \mathrm{ml})$ in $11 / 17(64.7 \%)$ subset 3 patients but not in any subset 1 or subset 2 patient $(P=0.0001)$.

The mean capillary number was $>9 / \mathrm{mm}$ in 41 patients, 9 to $7 / \mathrm{mm}$ in 24 patients and 6 to $4 / \mathrm{mm}$ in six patients. No statistical difference was found among these three subgroups in the prevalence of each clinical, preclinical functional and laboratory abnormality investigated (data not shown). Finally, none of the investigated clinical (puffy fingers, arthritis), preclinical (DLCO/forced vital capacity reduction, E/A ratio inversion), or serological
(SSc marker autoantibodies, ICTP, sE-selectin, sIL-2R $\alpha$ ) parameters was associated with the disease duration, as evaluated by RP onset.

\section{Discussion}

To our knowledge, this is the first study to systematically explore clinical and preclinical organ involvement and circulating activation markers in early SSc patients subdivided into three subsets, depending on the presence of both marker autoantibodies and NVC scleroderma pattern or either of these features.

Firstly, we found that our early SSc patients, which were enrolled by two distinct clinical units, were similar in sex and age distribution and disease duration, but differed in terms of clinical features and serum levels of 


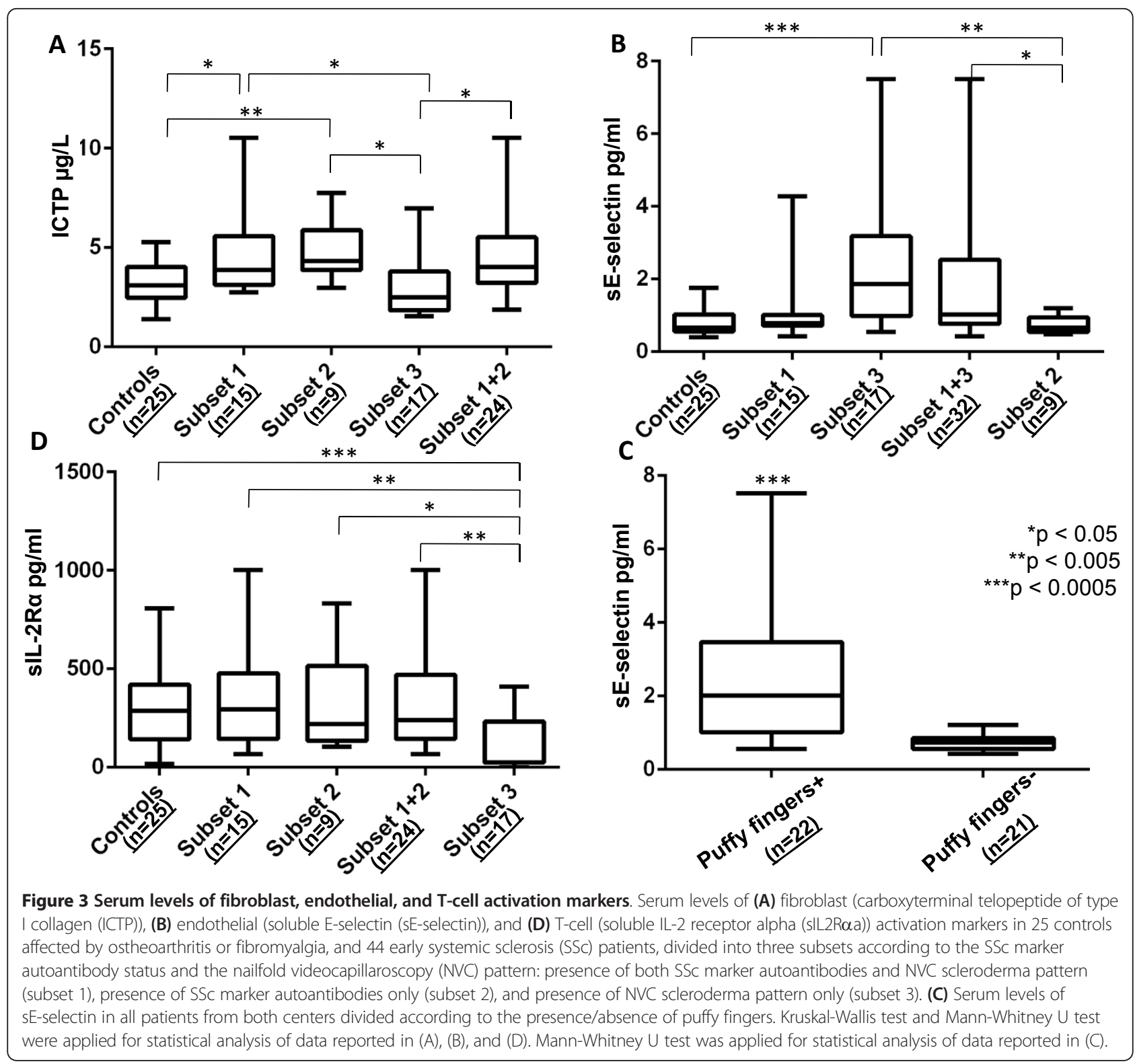

fibroblast, endothelial, and T-cell activation markers at presentation.

Given the different target patients of the two units, it is not surprising that a higher prevalence of SSc marker autoantibody-positive patients was enrolled by the Rheumatology Unit whereas a higher prevalence of RP patients presenting with puffy fingers was enrolled by the Angiology Unit. These differences are in line with results reported in patients with definite SSc by Walker and colleagues, who analyzed data entered into the European League Against Rheumatism (EULAR) Scleroderma Trials and Research database and found that differences among patients from various centers depend mainly on the clinical attitude of the respective medical team [16]. Notwithstanding this possible center-related recruitment bias, in our study early SSc patients with marker autoantibodies had a higher prevalence of impaired DLCO and higher circulating levels of ICTP irrespective of an NVC scleroderma pattern (megacapillaries and/or avascular areas). Similarly, early SSc patients with an NVC scleroderma pattern had a higher prevalence of puffy fingers and higher circulating levels of E-selectin irrespective of circulating marker autoantibodies, the two features being closely related each other. These associations have never previously been searched for. Moreover, four out of the 14 Rheumatology Unit patients with DLCO $<80 \%$ of the predicted value already presented mild alterations of lung anatomy as detected by high-resolution computed tomography.

In this context, it is noteworthy that an impaired DLCO is considered the first alteration in SSc interstitial 
lung disease [14,17]. Increased ICTP levels have been reported in SSc patients [18] and suspected SSc patients (that is, patients not meeting the preliminary 1990 ACR criteria for SSc) [4]. Moreover, elevated ICTP levels have been found to correlate with modified Rodnan skin score and to be associated with a diffuse disease and a reduced pulmonary function [19]. Given these observations, it is conceivable that early SSc patients with SSc marker autoantibodies are at higher risk of developing fibrotic organ complications.

Puffy fingers and RP are considered the main clinical features of the undifferentiated connective tissue syndrome [20]. sE-selectin, which reflects endothelial activation, has been reported to be increased in patients with definite SSc [21]. Our study demonstrates that puffy fingers are detectable in early SSc patients, mainly in those with an NVC scleroderma pattern and increased sEselectin levels. Serum IL-2R $\alpha$ levels, which reflect the activation of the adaptive immune response through T-cell recruitment, have been found to be strongly associated with mortality and inversely correlated to disease duration in SSc patients with diffuse disease [22]. Intriguingly, we found that sIL-2R $\alpha$ levels were strikingly lower in early SSc patients who were marker autoantibodynegative. This result could indicate a lack of T-cell recruitment in the peripheral blood of autoantibodynegative early SSc patients.

Taken together, the results of our study seem to suggest that the major cellular pathways involved in the pathogenesis of SSc, as evaluated by serum concentration of relevant activation markers, differ among early patients at presentation, in relation to autoantibody and microvascular status. Nevertheless, the asynchronous appearance of autoantibody positivity and capillaroscopic alterations has been reported in two recent papers. First, Englert and colleagues described the case of one patient in whom anti-Scl-70 positivity, associated with fatigue, weight loss and puffy fingers, predated the appearance of RP and the development of capillaroscopic abnormalities [23]. Second, Moinzadeh and colleagues reported the late appearance of serum autoantibodies in three patients with an NVC scleroderma pattern at admission [24]. Whether SSc marker autoantibody positivity or the presence of an NVC scleroderma pattern really do identify the dominating activation of a given cellular pathway at the disease onset in early SSc patients can only be determined by investigating circulating and tissue markers of cell activation contemporaneously.

Our study has some limitations. First, it might be affected by a differential verification bias because the patients were examined by clinicians with different medical expertise. To reduce this possible bias, however, capillaroscopic images and sera were made anonymous and centralized to the
Rheumatology Unit. Capillaroscopic images were blindly evaluated by one investigator experienced in NVC (MI); sera were blindly analyzed in duplicate by one investigator experienced in immunoassays (SV). Moreover, the clinical (puffy fingers) and preclinical (DLCO and E/A ratio) parameters used to assess both series of patients are well defined and routinely applied in clinical practice. Second, given the small number of patients, our conclusions may be susceptible to be changed by future studies on large number of patients, such as the ongoing Very Early Diagnosis of Systemic Sclerosis (VEDOSS) project $[25,26]$. In addition, one must underline that using the new ACR/ EULAR classification criteria presented at the ACR 2012 meeting $[27,28]$, a number of our early SSc patients could already meet the criteria for SSc at presentation. Indeed, in a post-hoc analysis 10/71 (14.1\%) patients met the new EULAR/ACR classification criteria for SSc and belonged to subset 1 (that is, patients with both SSc marker autoantibodies and capillaroscopic abnormalities). These patients met the new criteria because of the presence of puffy fingers along with SSc marker autoantibodies and the NVC scleroderma pattern. However, our major findings - that is, early SSc patients with SSc marker autoantibody have higher ICTP levels, regardless of the NVC pattern; and early SSc patients with an NVC scleroderma pattern have a higher prevalence of puffy fingers and higher circulating levels of E-selectin, regardless the autoantibody profile, the two features being closely related each other - did not differ significantly when we excluded these 10 patients from the analysis (see Additional files 1, 2, 3 and 4).

In conclusion, we found that early SSc patients show distinct clinical and preclinical features, and distinct circulating markers of cellular activation at presentation, according to their autoantibody and capillaroscopic status. Longitudinal large studies are required to verify whether the pathophysiological (cellular activation markers), functional (DLCO) and clinical (puffy fingers) differences in early SSc subsets that we detected in a small yet well characterized cohort really do translate into the development of different disease patterns over time.

\section{Additional material}

Additional file 1: Table S1 presenting epidemiologic, laboratory and capillaroscopic features in patients who did not meet EULAR/ACR classification criteria, and figure legends for Figures S1, S2, and S3.

Additional file 2: Figure S1 showing the prevalence of puffy fingers, arthritis and NVC scleroderma pattern in patients who did not meet EULAR/ACR classification criteria.

Additional file 3: Figure S2 showing the prevalence of DCLO impairment and autoantibody positivity in patients who did not meet EULAR/ACR classification criteria.

Additional file 4: Figure S3 showing serum levels of ICTP, sEselectin, sIL2Roa and sE-selectin in patients who did not meet EULAR/ACR classification criteria. 


\section{Abbreviations}

ACR: American College of Rheumatology; DLCO: diffusing lung capacity for carbon monoxide; E/A ratio: early/(atrial) late ventricular filling velocity ratio; ELISA: enzyme-linked immunosorbent assay; EULAR: European League Against Rheumatism; ICTP: carboxyterminal telopeptide of type I collagen; NVC: nailfold videocapillaroscopy; RP: Raynaud's phenomenon; sE-selectin: soluble E-selectin; sIL-2Ra: soluble IL-2 receptor alpha; SSc: systemic sclerosis.

\section{Competing interests}

The authors declare that they have no competing interests.

\section{Authors' contributions}

GV conceived, designed and coordinated the study, and drafted and critically revised the manuscript. AM acquired clinical data, performed nailfold videocapillaroscopy and participated in the design of the study. GC acquired clinical data, performed the statistical analysis, and participated in the design of the study and in drafting the manuscript. SV performed all immunoassays, the statistical analysis, and the post-hoc analysis, and drafted and critically revised the manuscript. Ml performed nailfold videocapillaroscopy and participated in the acquisition of data. FB performed autoantibody testing and routine biochemical analysis. CS and AC performed and analyzed lung function tests. DC and GMDM performed and analyzed B-mode echocardiography. SC and FV performed and analyzed chest X-ray scans. AS participated in the design of the study and critically revised the manuscript. All authors read and approved the final manuscript.

\section{Acknowledgements}

The study was supported by the Italian Foundation for Arthritis Research.

\section{Authors' details}

'Rheumatology Unit, Second University of Naples, via Pansini 5, 80131 Naples, Italy. ${ }^{2}$ Angiology Unit, Sandro Pertini Hospital, via dei Monti Tiburtini 385, 00157 Rome, Italy. ${ }^{3}$ Clinical Biochemistry Service, Sandro Pertini Hospital, via dei Monti Tiburtini 385, 00157 Rome, Italy. ${ }^{4}$ Respiratory Physiopathology Unit, ASL-SA1, via Santoriello 2, Cava dei Tirreni (SA), Italy. ${ }^{5}$ Pneumology Unit, Sandro Pertini Hospital, via dei Monti Tiburtini 385, 00157 Rome, Italy. ${ }^{6}$ Internal Medicine Unit, Second University of Naples, via Pansini 5, 80131 Naples, Italy. ${ }^{7}$ Cardiology Unit, Sandro Pertini Hospital, via dei Monti Tiburtini 385, 00157 Rome, Italy. ${ }^{8}$ Radiology, Radiotherapy and Nuclear Medicine Unit, Second University of Naples, Piazza Miraglia 5, 80131 Naples, Italy. ${ }^{9}$ Radiology Unit, Sandro Pertini Hospital, via dei Monti Tiburtini 385, 00157 Rome, Italy.

Received: 6 November 2012 Revised: 20 February 2013

Accepted: 29 May 2013 Published: 29 May 2013

\section{References}

1. LeRoy EC, Medsger TA Jr: Criteria for the classification of early systemic sclerosis. J Rheumatol 2001, 28:1573-1576.

2. Koenig M, Joyal F, Fritzler MJ, Roussin A, Abrahamowicz M, Boire G, Goulet JR, Rich E, Grodzicky T, Raymond Y, Senécal JL: Autoantibodies and microvascular damage are independent predictive factors for the progression of Raynaud's phenomenon to systemic sclerosis. A twentyyear prospective study of 586 patients with validation of proposed criteria for early systemic sclerosis. Arthritis Rheum 2008, 58:3902-3912.

3. Valentini G, Cuomo G, Abignano G, Petrillo A, Vettori S, Capasso A, Cozzolino D, Del Genio G, Santoriello C: Early systemic sclerosis: assessment of clinical and pre-clinical organ involvement in patients with different disease features. Rheumatology (Oxford) 2011, 50:317-323.

4. Valentini G, Vettori S, Cuomo G, ludici M, D'Abrosca V, Capocotta D, Del Genio G, Santoriello C, Cozzolino D: Early systemic sclerosis: short-term disease evolution and factors predicting the development of new manifestations of organ involvement. Arthritis Res Ther 2012, 14:R188.

5. Subcommittee for Scleroderma Criteria of the American Rheumatism Association Diagnostic and Therapeutic Criteria Committee: Preliminary criteria for classification of systemic sclerosis (scleroderma). Arthritis Rheum 1980, 23:581-590.

6. LeRoy EC, Medsger TA Jr: Raynaud's phenomenon: a proposal for classification. Clin Exp Rheumatol 1992, 10:485-488.

7. Sebastiani M, Manfredi A, Vukatana G, Moscatelli S, Riato L, Bocci M, Iudici M, Principato A, Mazzuca S, Del Medico P, De Angelis R, D’Amico R, Vicini $R$, Colaci $M$, Ferri C: Predictive role of capillaroscopic skin ulcer risk index in systemic sclerosis: a multicentre validation study. Ann Rheum Dis 2012, 71:67-70.

8. Maricq HR: Widefield capillary microscopy: technique and rating scale for abnormalities seen in scleroderma and related disorders. Arthritis Rheum 1981, 24:1159-1165.

9. Scussel-Lonzetti L, Joyal F, Raynauld J-P, Roussin A, Rich E, Goulet JR, Raymond Y, Senécal JL: Predicting mortality in systemic sclerosis. Analysis of a cohort of 309 French Canadian patients with emphasis on features at diagnosis as predictive factors for survival. Medicine (Baltimore) 2002, 81:154-167.

10. Caramaschi P, Canestrini S, Martinelli N, Volpe A, Pieropan S, Ferrari M, Bambara LM, Carletto A, Biasi D: Scleroderma patients nailfold videocapillaroscopic patterns are associated with disease subset and disease severity. Rheumatology 2007, 46:1566-1569.

11. Nishimura RA, Miller FA Jr, Callahan MJ, Benassi RC, Seward JB, Tajik AS: Doppler echocardiography: theory, instrumentation, technique and application. Mayo Clin Proc 1985, 60:321-343.

12. Maione S, Cuomo G, Giunta A, Tanturri de Horatio L, La Montagna G, Manguso F, Alagia I, Valentini G: Echocardiographic alterations in systemic sclerosis. A longitudinal study. Semin Arthritis Rheum 2005, 34:721-727.

13. Ogilvie CM, Forster RE, Blakemore WS, Morton JW: A standardized breath holding technique for clinical measurement of the diffusing capacity of the lung for carbon monoxide. J Clin Invest 1957, 36:1-17.

14. Steen VD, Owens GR, Fino GJ, Rodnan GP, Medsger TA: Pulmonary involvement in systemic sclerosis (scleroderma). Arthritis Rheum 1985, 28:759-767.

15. Kazerooni EA, Martinez FJ, Flint A, Jamadar DA, Gross BH, Spizarny DL, Cascade PN, Whyte RI, Lynch JP, Toews G: Thin-section CT obtained at $10-\mathrm{mm}$ increments versus limited three-level thin-section $C T$ for idiopathic pulmonary fibrosis: correlation with pathologic scoring. Am J Roentgenol 1997, 169:977-983.

16. Walker UA, Tyndall A, Czirják L, Denton CP, Farge-Bancel D, KowalBielecka O, Müller-Ladner U, Matucci-Cerinic M, EUSTAR co-authors: Geographical variation of disease manifestations in systemic sclerosis: a report from the EULAR Scleroderma Trials and Research (EUSTAR) group database. Ann Rheum Dis 2009, 68:856-862.

17. Wells AU, Steen V, Valentini G: Pulmonary complications: one of the most challenging complications of systemic sclerosis. Rheumatology (Oxford) 2009, 48(Suppl 3):40-44.

18. Scheja A, Wildt M, Wollheim FA, Akesson A, Saxne T: Circulating collagen metabolities in systemic sclerosis. Differences between limited and diffuse form and relationship with pulmonary involvement. Rheumatology 2000, 39:1110-1113.

19. Allanore $Y$, Borderie D, Lemaréchal $H$, Cherrau B, Ekindjian OG, Kahan A: Correlation of serum collagen I carboxyterminal telopeptide concentrations with cutaneous and and pulmonary involvement in systemic sclerosis. J Rheumatol 2003, 30:68-73.

20. LeRoy EC, Maricq HR, Kahaleh MB: Undifferentiated connective tissue syndromes. Arthritis Rheum 1980, 23:341-343.

21. Ihn H, Sato S, Fujimoto M, Takehara K, Tamaki K: Increased serum levels of soluble vascular cell adhesion molecule-1 and E-selectin in patients with systemic sclerosis. Br J Rheumatol 1998, 37:1188-1192.

22. Degiannis D, Seibold JR, Czarnecki M, Raskova J, Raska K Jr: Soluble interleukin-2 receptors in patients with systemic sclerosis. Clinical and laboratory correlations. Arthritis Rheum 1990, 33:375-380.

23. Englert H, Champion D, Wu JC, Giallussi J, McGrath M, Manolios N: Antitopoisomerase antibody positivity predates nailfold capillaroscopy abnormalities in scleroderma. Postulated classification of 'prescleroderma'. Intern Med J 2011, 41:197-199.

24. Moinzadeh Pia, Nihtyanova SI, Howell K, Ong VH, Denton CP: Impact of hallmark autoantibody reactivity on early diagnosis of scleroderma. Clin Rev Allergy Immunol 2012.

25. Matucci-Cerinic M, Allanore Y, Czirják L, Tyndall A, Müller-Ladner U, Denton C, Valentini G, Distler O, Fligelstone K, Tyrrel-Kennedy A, Farge D, Kowal-Bielecka O, van den Hoogen F, Cutolo M, Sampaio-Barros PD, Nash P, Takehara K, Furst DE: The challenge of early systemic sclerosis for the EULAR Scleroderma Trial and Research group (EUSTAR) community. It is time to cut the Gordian knot and develop a prevention or rescue strategy. Ann Rheum Dis 2009, 68:1377-1380.

26. Avouac J, Fransen J, Walker UA, Riccieri V, Smith V, Muller C, Miniati I,

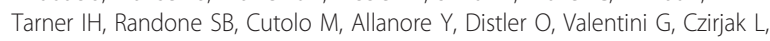


Müller-Ladner U, Furst DE, Tyndall A, Matucci-Cerinic M, EUSTAR Group: Preliminary criteria for the very early diagnosis of systemic sclerosis: results of a Delphi Consensus Study from EULAR Scleroderma Trials and Research Group. Ann Rheum Dis 2011, 70:476-481.

27. Fransen J, Johnson SR, van den Hoogen F, Baron M, Allanore Y, Carreira PE, Czirják L, Denton CP, Distler O, Furst DE, Gabrielli A, Herrick A, Inanc M, Kahaleh B, Kowal-Bielecka O, Medsger TA Jr, Mueller-Ladner U,

Riemekasten G, Sierakowski S, Valentini G, Veale D, Vonk MC, Walker U, Chung L, Clements PJ, Collier DH, Csuka ME, Jimenez S, Merkel PA, Seibold JR, et al: Items for developing revised classification criteria in systemic sclerosis: results of a consensus exercise. Arthritis Care Res 2012, 64:351-357.

28. Johnson SR, Fransen J, Khanna D, Baron M, van den Hoogen F, Medsger TA $\mathrm{Jr}$, Peschken CA, Carreira PE, Riemekasten G, Tyndall A, Matucci-Cerinic M, Pope JE: Validation of potential classification criteria for systemic sclerosis. Arthritis Care Res 2012, 64:358-367.

doi:10.1186/ar4236

Cite this article as: Valentini et al:: Early systemic sclerosis: marker autoantibodies and videocapillaroscopy patterns are each associated with distinct clinical, functional and cellular activation markers. Arthritis Research \& Therapy 2013 15:R63.

\section{Submit your next manuscript to BioMed Central} and take full advantage of:

- Convenient online submission

- Thorough peer review

- No space constraints or color figure charges

- Immediate publication on acceptance

- Inclusion in PubMed, CAS, Scopus and Google Scholar

- Research which is freely available for redistribution

Submit your manuscript at www.biomedcentral.com/submit 